\title{
Diskussion zum Vortrag von Gunnar Duttge
}

\author{
Leitung: OKKO BEHRENDS
}

\section{BEHRENDS:}

Wir haben jetzt eine gute halbe Stunde Zeit für die Diskussion. Ich darf gleich zu Anfang Herrn Duttge dafür danken, dass er sein Thema so prägnant an den beiden Menschenbildern, an dem Gegensatz zwischen dem privatautonomen und dem sozialen Menschenbild profiliert hat, an einem Spannungsverhältnis, das von der antiken Philosophie in der Schwebe gehalten wurde. Dagegen scheint in der Neuzeit die Ansicht, die den Menschen als soziales Element der Gesellschaft sieht, mit einer gewissen Einseitigkeit im Vordringen zu sein. Das hat unter anderem den Gedanken der défense sociale immer stärker in das Zentrum treten lassen, und Herr Duttge hat uns eindrucksvolle Beispiele dafür gegeben, was das bedeutet, insbesondere für die Wertungen, die hinter den Sanktionen des Strafrechts stehen.

Ich möchte im Zusammenhang damit eine den Wert des Individuums betreffende Frage aufwerfen, die mich schon seit frühester Zeit bewegt, seit einer Diskussion, die ich als Hilfskraft mit dem damaligen Institutsassistenten hatte, und auf die auch zum Teil die Anregung zu diesem Symposion zurückgeht. Sie betraf die Frage, ob folgende Aussage erlaubt ist: Ich bin ein entschiedener Gegner der Todesstrafe, bin aber gleichzeitig davon überzeugt, dass es nach wie vor moralisch todeswürdige Verbrechen gibt. Ein solche Satzfolge will unterscheiden: Es ist ein positives Ergebnis der Humanisierung unseres Strafvollzuges, dass die Todesstrafe abgeschafft ist. Aber es gibt immer noch Taten, bei denen man dem Täter sagen kann und muss: Du musst dir klar machen, dass du moralisch nach wie vor durchaus den Tod verdient hättest und deine Tat nicht dadurch weniger verwerflich wird, dass du in einem humanen Rechtsstaat lebst. Mein Gesprächspartner war damals entschieden gegen die Zulässigkeit einer solchen Rede. Ich meine demgegenüber: Man muss dem verbreiteten Fehlschluss entgegentreten, dass die Humanisierung des Strafvollzugs auch das moralisch abwertende Urteil über die Tat mildert. Man sollte die Spannung zwischen Recht und mitmenschlicher Ethik aushalten und das moralische Werturteil nicht davon bestimmen lassen, dass wir als humanes Gemeinwesen weder die Todesstrafe noch eine sinnlos gewordene Verwahrstrafe wollen. Das Unwerturteil, das hinter dem Tötungsverbot steht, sollte nicht verblassen. Die gegenteilige Entwicklung hat gewiss mit den massenhaften Erscheinungen staatlichen, organisierten, sich als Töten aus nicht niedrigen Beweggründen verstehenden Mordens zu tun und der Art seiner gerichtlichen 
Bewältigung. Das hat offensichtlich zu einer gewissen Abstumpfung der Empfindungen und zur Relativierung grundlegender Unwerturteile geführt. Der derzeitige Präsidentschaftskandidat der USA, der president-elect, für unsere global gewordene Kultur gewiss eine repräsentative Figur, tritt demgegenüber sogar, was ich nicht billige, für die Todesstrafe ein. Er will über das Recht der Gesellschaft die Möglichkeit bewahren, in sehr schlimmen Fällen, Mord an Kindern, die Verwerflichkeit der Tat auf diese Weise auszudrücken. Die politische Herkunft dieser Stimme ist bedenkenswert. Das Strafrecht ist immer in Gefahr, durch professionelle Gewöhnung die existentielle, humane und moralische Dimension seines Gegenstands verblassen zu lassen. Daher kann man vielleicht unsere Tagung, in der ja die Strafrechtler nicht in der Mehrheit sind, unter das Motto stellen: Das Strafrecht ist zu ernst, als dass man es nur den Strafrechtlern überlassen dürfte. Es braucht jedenfalls eine Ethik, die zwischen Humanisierung des Strafens und dem fundamentalen Unwert des Tötens zu unterscheiden vermag. Erinnern möchte ich in dem Zusammenhang auch an die Worte von Frau Schumann bei der Begrüßung und Eröffnung. Sie haben bestätigt, dass der strafende Sozialstaat, das Thema unserer Zusammenkunft, eigentlich ein Oxymoron ist. Denn der Sozialstaat versteht sich als Helfer dessen, mit dem er zu tun bekommt. Daher findet sich in der Literatur die Forderung, dass die Sozialpolitik das Strafrecht ersetzen, dass an die Stelle der Kriminalpolitik die Sozialpolitik treten sollte.

Also: Wir haben viele Gesichtspunkte und ich darf die Diskussion eröffnen. Wer meldet sich zuerst? Herr Schreiber, bitte.

\section{SCHREIBER:}

Herr Duttge, Ihr Vortrag war hochgescheit und sehr belesen. Nur, er lässt mich einigermaßen hilflos, und es stellen sich mir viele Fragen. Wenn ich Sie recht verstanden habe, meinen Sie, dass Basis des Strafens immer Ausgleich zugerechneter Schuld sein muss. Sie mögen Generalprävention weniger, wollen dann aber doch noch ein kräftiges Stück Spezialprävention draufsetzen, sonst wäre das sozialstaatlich unsinnig und würde den Staat, wenn es nur um Ausgleich von Schuld ginge, eigentlich überheben, wenn ich Sie recht verstanden habe. Aber machen Sie nicht genau das, was JAKOBS den anderen vorwirft? Sie machen eine Mischung von allen möglichen Gesichtspunkten, freilich mit einem extrem deutlichen Akzent auf dem Gesichtpunkt der $\mathrm{Ge}-$ rechtigkeit. Und Sie bleiben dabei stehen, dass Sie sagen, das müsse ausgeglichen werden. Das soll offenbar die Basis bilden. Mir fehlt die Rolle der Maßregel. Welche Rolle spielt die Maßregel neben der Strafe, die Gefährdung auch ohne Verschulden, gerade ohne Verschulden, wenn es so etwas überhaupt gibt wie Verschulden, denn unsere neuen Hirnforscher sagen, das gäbe es ja alles gar nicht. Das haben Sie ein bisschen am Rande angefasst. Wie passt sich die Maßregel ein? Was übernimmt die Maßregel von der Strafe? Und wie steht die Maßregel zur Strafe? Denn die Einführung der Maßregel ist in unser Strafrecht wohl auch geschehen, einfach aus der Ratlosigkeit über den Stel- 
lenwert des Schuldausgleiches und der Prävention. Können Sie dazu noch etwas sagen?

BEHRENDS:

Ich glaube, die Frage ist klar. Es geht um Zweispurigkeit. Herr Duttge, möchten Sie gleich antworten? Das ist für die Diskussion lebhafter, als wenn wir sammeln.

\section{DUTTGE:}

Ja, noch einmal ganz grundsätzlich zu meinem Anliegen: Es geht mir im Kern darum, ein konsistentes Menschenbild zu entwickeln, das Grundlage nicht nur für die Frage sein kann, wann und unter welchen Voraussetzungen ein Straftäter schuldhaftes Unrecht begangen hat, sondern ein Menschenbild, das ebenso - in unveränderter Gestalt - auch im Rahmen der Strafzumessung und Strafvollstreckung Geltung beanspruchen kann; ein solchermaßen einheitliches, gleichsam im übergreifenden Zusammenhang innerhalb des gesamten strafrechtlichen Rechtsgangs gültiges Menschenbild vermag ich bisher nicht zu entdecken. Insofern geht mein Bemühen gerade nicht dahin, ein „Potpourri“ anzurühren, also einer je nach Bedarf wechselnden Akzentuierung des Menschenbildes das Wort zu reden, was aus meiner Sicht eher Ausdruck von Ratlosigkeit, eine Kapitulation vor der Aufgabe wäre, ein rationales Strafbegründungs- und Strafrechtsanwendungskonzept zu präsentieren. Konkreter gefasst geht es mir somit um ein Bild vom Menschen, das diesen nicht nur im Sinne einer rein liberalen Philosophie als „,in Freiheit gesetztes Wesen“ begreift, sondern auch in seiner sozialen Existenz, so dass der von mir hervorgehobene resozialisierende Einschlag hoheitlicher Strafgewalt unverzichtbar ist. Sie haben, lieber Herr Schreiber, dies als Basis der Strafe bezeichnet; gerade das ist es aber nach meiner Überzeugung nicht; vielmehr hat das Anliegen des strafenden Gesetzgebers ausschließlich die Sanktionierung eines schuldhaft verübten Rechtsbruchs zum Gegenstand. Anlass und Grund des staatlichen Strafens ist also einzig die vorausgegangene Straftat; insofern hat die Strafe notwendig eine retributive Zielrichtung. Inwieweit sie sich im konkreten Fall dann auch tatsächlich rechtfertigen lässt, eine Frage, die missverständlich als solche der Strafzwecke verhandelt wird, ist in Wahrheit eine solche, die sich nur aufgrund einer Gerechtigkeitstheorie beantworten lässt.

\section{BEHRENDS:}

Vielen Dank. Es gibt die Suche nach dem homogenen Menschenbild im Strafrecht. Es gibt aber auch die Zweispurigkeit, die hinter dem Nebeneinander von Strafe und Maßregeln der Sicherung und Besserung steht. Herr Frisch.

FRISCH:

Herr Duttge, Sie haben Ihr eigenes Konzept durchscheinen lassen. Ich stimme Ihnen insoweit in vielem zu. Es ist deutlich geworden, dass Sie stark mit dem Gedanken des Schuldausgleichs, also mit einer schuldorientierten Strafe, sympathisieren. Damit bleibt natürlich die Frage nach dem Stellenwert der Präven- 
tion zu beantworten. Ihr eigenes Modell läuft insoweit, wenn ich es richtig verstanden habe, darauf hinaus, dass eine Art Rahmenmodell der Schuld der Praxis einen Spielraum geben soll, in dem dann auch die Prävention berücksichtigt wird - freilich nur ein bisschen, denn alle Bedürfnisse der Prävention lassen sich in diesem Rahmen nicht befriedigen. Ich frage mich bei dieser Art der Strafdiskussion immer eines: Leidet die ganze Diskussion nicht zu sehr darunter, dass wir einfach von einem traditionellen Institut (der Strafe) ausgehen und dann fragen, wie gewisse unterschiedliche Staatsaufgaben (nämlich die Aufrechterhaltung der Geltungskraft von Normen und die reale Prävention) in diesem Institut zusammenkommen, sich in dieses Institut gewissermaßen einfügen? Wäre es nicht sehr viel sinnvoller, man würde von vornherein von den Staatsaufgaben ausgehen, nämlich sagen, dass wir auf der einen Seite die Aufgabe haben, eine Normenordnung zu bestätigen, deren Geltungskraft durch die begangene Straftat erschüttert worden ist. Den Maßstab dafür bildet das Gewicht des verschuldeten Normbruchs. Diese Bestätigung der verletzten Rechtsordnung bildet etwas, worauf wir angewiesen sind. Das ist im Grunde die Aufgabe der Strafe.

Es gibt aber auch noch eine andere Staatsaufgabe. Und diese lässt sich nicht einfach in der zuvor aufgabenorientiert bestimmten Strafe unterbringen, jedenfalls nicht insgesamt. Ich meine den Schutz der Gesellschaft vor drohenden Straftaten, das, was Sie, Herr Behrends, vorhin als défense sociale bezeichnet haben. Diese Aufgabe muss, wenn sie anderes als die Bedürfnisse der Normbestätigung fordert, in einem anderen Institut (z.B. so genannten Maßregeln) verwirklicht werden.

DUTTGE:

Gerade wenn man die Strafe als ganz eigenen, spezifischen Typus hoheitlichen Handelns von den sonstigen Staatsaufgaben deutlich genug unterscheiden will, muss man unweigerlich auf die Idee des gerechten Tatausgleichs als zentrale Sinngebung zu sprechen kommen. Denn in der Tat leidet die Strafzweckdebatte seit langem darunter, dass alles zusammengemischt, also der „Zweck“ des Strafens mit den sonstigen Staatsaufgaben vermengt wird und damit das Spezifikum des Strafens verloren geht. Das machen sich übrigens manche Vertreter der Neurowissenschaften zunutze, indem zur Begründung des von manchem geforderten Übergangs von einem Schuldstraf- zu einem auf Prävention und Erziehung gerichteten Maßregelrecht auf den Stand der Strafzweckdebatte verwiesen wird, in der selbst entfernte Anleihen an den Vergeltungsgedanken als „,barbarisch“ und ganz und gar indiskutabel gelten. Erst wenn aber zwischen Grund und Notwendigkeit des Strafens getrennt wird, zeigt sich, was Strafen bedeutet und was nicht. Die Frage der Notwendigkeit des Strafens ist nämlich eine ganz andere und kann etwa dazu führen, dass das Ausmaß der Intervention in manchen Fällen gemildert oder durch Einbeziehung weiterer Zwecke neben jenem des Schuldausgleichs erweitert werden kann. Insofern ist grundsätzlich nichts dagegen einzuwenden, wenn mit der Tatvergeltung unter Um- 
ständen die Verhängung einer Maßregel der Besserung und Sicherung verknüpft wird. Verglichen mit der Strafe verfolgt eine solche Maßregel aber einen ganz anderen Zweck, ist etwas anderes. Mit ihr wird ein weiteres, vom Strafzweck strikt zu trennendes Ziel hoheitlicher Intervention hinzugenommen, das man in letzter Konsequenz im Streben nach Gefahrenvorsorge zum Wohle der Allgemeinheit, gegebenenfalls auch nach Implementierung notwendiger Fürsorge zum Wohle des therapiepflichtigen Straftäters erkennen kann. Dadurch bleibt aber der Kern dessen, was Strafe bezweckt und trägt, unberührt.

\section{BEHRENDS:}

Herr Naucke.

NAUCKE:

Herr Duttge, ich habe Ihnen mit Interesse und wirklichem Gewinn zugehört. Es ist ein sumpfiges Feld, über das man redet, und ich habe den Eindruck, Sie haben durch diesen Sumpf einen ganz festen Pfad gelegt, auf dem Sie auch auf der anderen Seite des Sumpfes angekommen sind. Das finde ich in der gegenwärtigen Debatte, die unübersichtlich ist, eine große Leistung, und für jemanden, der Ihnen zuhört, eine zusätzliche Information. Meine Frage an Sie bezieht sich auf Überlegungen, die schon angestellt worden sind. Machen Sie sich die Sache nicht zu einfach gegenwärtig, oder begrenzen Sie sie nicht so, dass sie dann lösbar wird, indem Sie nur von der Strafe reden? Wir haben ein Straf- und Maßregelgesetzbuch. Das muss man den Studenten immer wieder sagen. Das steht in einem Gesetz. Und damit ist es nicht zu Ende. Wir haben einen nahtlosen Übergang von Straf- und Maßregeln zu den Ordnungswidrigkeiten, die heute eine Stelle einnehmen, die man sich früher bei der Strafe nie vorstellen konnte. Wir haben Strafe, Maßregel, Ordnungswidrigkeiten, dann das Polizeirecht, das immer mehr auf der Seite des Strafrechts sich ansiedelt und strafrechtliche Linien zu übernehmen sich anschickt. Und wir haben, das gehört mit dazu, das Sozialrecht. Wenn ich mir im Sozialgesetzbuch XII ansehe, was dort über die sozial Abweichenden steht, dann hat man tatsächlich heute ein nicht trennbares Kontinuum von der Strafe bis zur Sozialfürsorge. Und die Frage, die wir uns stellen müssen, ist sicher die nach dem menschengerechten Strafen, da stimme ich Ihnen zu und wäre auch bereit, Ihre Lösungen mit Ihnen in Ihrer Richtung zu debattieren. Aber damit kommen wir nicht weiter. Es geht um menschengerechte soziale Kontrolle. Und die kann man nicht in einzelne Schubladen teilen und sagen, das machen wir so, das machen wir so, das machen wir so. Dann machen wir es dem Gesetzgeber zu einfach. Ab damit in die Schublade, mit der ich am besten zurechtkomme, und so handelt er ja im Moment. Und schiebt dann Straftaten in die Maßregeln, Maßregeln in die Ordnungswidrigkeiten usw. Meine Frage ist: Lässt sich Ihr Ergebnis so verallgemeinern, dass es jede Form sozialer Kontrolle erfasst? 
BEHRENDS:

Vielen Dank. Herr Duttge.

DUTTGE:

Ich habe mich in der Tat, das sehen Sie ganz richtig, auf die Strafe im engeren Sinne und damit auf die sich mit ihr verbindende, nach meiner Überzeugung ganz spezifische, eigengeartete Zwecksetzung konzentriert. Das geschah natürlich sehr wohl vor dem Hintergrund, dass wir auch andere, insbesondere sozialstaatlich motivierte Bedürfnisse und Instrumente kennen. Ich sagte ebenso bereits, dass mitunter Kombinationsmöglichkeiten vorkommen. Das darf uns aber nicht dazu verleiten, die Sinngebung der jeweiligen Teile, soweit diese auch für sich stehen können, aus dem Blick zu verlieren. Deshalb lege ich groBen Wert darauf, zunächst zu verdeutlichen, was der spezifische Sinn des Strafens ist und was - davon geschieden - die anderen Interventionsmöglichkeiten bezwecken. Dass zuletzt alles aufgehen könnte in einen übergreifenden gemeinsamen ,Zweck“, der auch die Sinngebung staatlichen Strafens mit einbezöge, und infolgedessen dasjenige, was ich zum gerechten Schuldausgleich gesagt habe, an Geltungskraft verlöre, das vermag ich im Augenblick nicht zu erkennen. Eine solchermaßen ganzheitliche Betrachtung würde nur auf hohem Abstraktionsniveau das Eigentliche zum Verschwinden bringen. All die erwähnten Phänomene der modernen Sozialfürsorge stehen mit ihrer Zwecksetzung neben der strafenden Intervention und nicht als deren Ersatz bereit - solange die RADBRUCHsche Utopie von etwas Besserem als Strafrecht (und nicht nur von einem besseren Strafrecht) weiterhin Utopie ist.

\section{BEHRENDS:}

Vielen Dank. Die nächste Wortmeldung. Herr Loos, bitte.

LoOs:

Wenn ich Sie richtig verstanden habe, haben Sie in das Menschenbild zwar soziale Dimensionen einbezogen, Sie halten aber doch wohl als Grundlage eines Strafrechts, das im Wesentlichen Schuldausgleich zur Bestätigung von Normen betreiben soll, grundsätzlich an einem Menschenbild fest, das einen solchen Schuldvorwurf, der dann ausgeglichen werden muss, tragen kann. Woher haben Sie das? Wenn ich Sie richtig verstanden habe, haben Sie Art. 1 GG und die Metaphysik in Art. 1 GG angedeutet. Sind danach die Hirnforscher verfassungswidrig? Ich formuliere das jetzt mal sehr zugespitzt, aber ich glaube es ist sehr deutlich, was ich damit meine.

BEHRENDS:

Ein selbständiges Thema! Herr Duttge.

DUTTGE:

$\mathrm{Zu}$ diesem weit gespannten Thema nur in aller Kürze: Ich hatte am Ende meines Vortrages bereits angedeutet, dass wir es uns möglicherweise etwas zu leicht machen bei der Zuschreibung individueller Schuld. Ich sehe die Bedeu- 
tung dessen, was durch die moderne Hirnforschung an Erklärungen und vor allem an Forderungen vorgetragen wird, vor allem darin, dass wir kritisch reflektieren sollten, inwieweit der Mensch in mancherlei Hinsicht vielleicht doch einem Mehr an Zwängen und heteronomen Einflüssen ausgesetzt sein könnte, als wir dies gemeinhin anerkennen. Mit anderen Worten sollten wir es uns nicht so leicht mit der Schuldzuschreibung machen, bezogen auf ein häufig hochemotional geprägtes Tatgeschehen, in das sich der Täter oftmals regelrecht verstrickt hat. Darin sehe ich die zentrale Bedeutung der aktuellen Debatten, ungeachtet aller Kritik an den bekannten Experimenten und an den weit überschießenden Folgerungen, die hieraus zum Teil gezogen werden. So gehe ich in der Tat davon aus, dass die willensbestimmte Handlungsfreiheit durch die Neurowissenschaften nicht widerlegt ist, dass vielmehr hinsichtlich der altehrwürdigen Frage nach der „Willensfreiheit“ ein non-liquet besteht; die Rechtfertigungslast für die Forderung, ob die Rechtsordnung auf einem neuen Fundament errichtet werden muss, sehe ich dabei auf Seiten der Hirnforschung. Noch ein letztes Wort zur Frage nach Art. 1 GG und seinem metaphysischen Fundament: In der Tat findet sich hier die Fähigkeit zur lebensweltlichen und sittlichen Selbstbestimmung normativ verankert, mit der sich zugleich die Berechtigung verbindet, für die Folgen eines selbstbestimmten Verhaltens Verantwortung zuzuschreiben. Die gedankliche Grundlage für die Prämisse ist aber in letzter Konsequenz nicht beweisbar, sondern bloßes Postulat. In diesem Vorverständnis liegt der metaphysische Anteil begründet, der dem Institut der Strafe aber denknotwendig immanent ist. Nur muss das Rechtsideal im konkreten strafrechtlichen Kontext wiederum geerdet, d.h. auf die Bedingungen und Begrenzungen des täter- und tatbezogenen realen Lebensumfeldes bezogen werden. Hier muss sich die Inverantwortungnahme eines Menschen als „,Täter“ letztlich hinsichtlich ihrer Berechtigung bewähren.

BEHRENDS:

Herr Jehle.

JEHLE:

Drei kurze Fragen: Die erste Frage zielt auf die Schuld bzw. die Schwere der Schuld. Wenn man die Zitate des BGH nimmt, die sich mit der Schwere der Schuld auseinandersetzen, dann schimmert eigentlich immer ein Stück positiver Generalprävention durch. So heißt es z.B. bezüglich $₫ 17$ JGG, Jugendstrafe wegen Schwere der Schuld sei erforderlich, wenn es schlechthin unverständlich für das Rechtsempfinden wäre, dass diese Tat nicht durch einen Freiheitsentzug sanktioniert wird. Im Grunde genommen steht hinter der Vergeltungsidee das Vergeltungsbedürfnis. Und damit ist man schon wieder auf dem Weg zur positiven Generalprävention.

Die zweite Frage betrifft die tatproportionale Strafzumessung. Wenn man sich zur Findung des konkreten Strafmaßes nur auf das übliche Maß, also auf Konvention bezieht, wäre mir dies zu wenig. Ich würde fragen, kann man Schuld in ein Strafquantum übersetzen, mit welchen Kriterien kann man das 
übersetzen? In diesem Zusammenhang hieß es auch, die Strafen seien heute doch recht milde, human geworden. Wenn man den abstrakten Strafrahmen nimmt, dann ist das aber gar nicht so. Der einfache Diebstahl kann mit bis zu fünf Jahren Freiheitsstrafe bestraft werden, und das Ganze erscheint nur milde, weil die Strafpraxis beim einfachen Diebstahl nur noch das untere Sechstel ausschöpft und weil sie zwei Drittel aller kleinen Diebstähle überhaupt nicht pönalisiert, also letztlich gar nicht mehr wie Straftaten behandelt.

Und zur dritten Frage: Was mir gänzlich gefehlt hat, ist neben der Schuld und der Strafzumessung die Sanktionenwahl. Das ist doch das Entscheidende. Wir tun so, als ob 90 Tagessätze Geldstrafe, drei Monate Freiheitsstrafe ohne Bewährung und drei Monate Freiheitsstrafe mit Bewährung dasselbe seien. Das ist aber in der Lebenswirklichkeit etwas ganz Unterschiedliches, das sich nach spezialpräventiven Gesichtspunkten entscheidet. Gerade bei der Vollstreckung der ausgesetzten Freiheitsstrafe haben wir doch alle Elemente der Spezialprävention und der Resozialisierung. Und das ist jetzt die letzte Bemerkung, zu Herrn Schreiber: Das präventive Element wohnt ja nicht nur der Maßregel inne, sondern die Strafe selbst und insbesondere ihre Vollstreckung ist heute präventiv aufgeladen. Wollte man dies sauber trennen, bräuchte man ein vollkommen neues Strafrecht.

DUTTGE:

Darauf läuft es meines Erachtens auch hinaus: Das Präventionselement ist seiner materiellen Substanz nach stets Polizeirecht, nicht Strafrecht, gleichgültig, wo und wie es in Erscheinung tritt. Wir betreiben daher, wenn und soweit wir den Präventionsgedanken in den Vordergrund rücken, in Wahrheit Polizeirecht in strafrechtlichem Gewande. Zu jenem darüber hinaus erwähnten Aspekt der Sanktionenwahl: In der Tat ist auch dies eine zentrale und nicht zu vernachlässigende Frage innerhalb der Idee einer tatproportionalen Strafzumessung: Bei dieser kann es also mitnichten nur um die Höhe, sondern muss es selbstverständlich auch um die Art der Sanktionierung gehen. Das wäre also ein weiteres, noch der Vertiefung bedürftiges Thema, zu dem es aber ebenfalls schon manche Vorarbeiten gibt. Nur: Was bedeutet das konkret? Sind wir gehalten, eine direkte Äquivalenz herzustellen zwischen der Art der verhängten bzw. vollstreckten Sanktion und der begangenen Tat? Also, um ein Beispiel zu nennen, Prügelstrafe für Körperverletzungsdelikte, oder wenn man eine Abstraktionshöhe weiter den Typus der Rechtsgutsverletzung maßgeblich sein lässt: Ist der strafende Zugriff auf Eigentum oder Vermögen des Täters allein bei Diebstahls- und Betrugstaten begründbar, weil nur hier eine entsprechende, rechtsgutsspezifische Korrelation besteht? Und was machen wir mit Mördern? Es ist leicht zu sehen, dass wir einen solchen Zusammenhang spätestens an dieser Stelle kaum akzeptieren werden und dementsprechend bei der Frage nach der ,richtigen“ Sanktionswahl in erhebliche Schwierigkeiten geraten. Schließlich noch ein letztes Wort zur These: Keine Schuld ohne positive Generalprävention? Nein, das würde ich doch anders sehen. Natürlich schim- 
mert in der heutigen straftheoretischen Debatte in vielen Konzeptionen der Generalprävention verdeckt doch der Gedanke des vergeltenden Schuldausgleichs mehr oder weniger durch. Positive Generalprävention dient dann aber gleichsam nur als postmetaphysischer Ersatz für die eigentlich noch immer gemeinte Tatvergeltung; einen gegenläufigen Zusammenhang gibt es dagegen nicht. Die Bedürfnisse der Allgemeinheit sind bereits in der Strafandrohung, also in der generellen Bewertung und tatbestandlichen Beschreibung möglicher Straftaten eingegangen. Sie haben jedoch nur mittelbare Bedeutung, gleichsam als Hintergrundfolie, wenn es um den konkreten Rechtsgang gegen einen konkret Beschuldigten geht; die Wahl jener Person, die mit den Mitteln des Strafrechts in Verantwortung genommen wird, entscheidet sich nicht nach Präventionsbedürfnissen, sondern danach, ob dieser Person die Tat auch als schuldhaft begangene zugerechnet werden kann, also allein anhand der ihr zugeschriebenen Schuld. Im Rahmen der Strafvollstreckung mögen sich dann mit der Ahndung dieser Straftat zugleich Präventionsbedürfnisse mit Blick auf eine hinzukommende besondere Gefährlichkeit dieses Straftäters verbinden, die eine prophylaktische Intervention vor Begehung künftiger Straftaten gebieten kann nach dem Motto: ,Jetzt haben wir den Täter schon in unserer Gewalt, dann prüfen wir, wenn er schon einmal straffällig geworden ist, ob und inwieweit er auch für die Begehung weiterer Straftaten anfällig ist, bevor wir ihn wieder herauslassen. " Das ist aber ein hinzutretendes und in seiner Zielsetzung gänzlich anders geartetes Motiv der Rechtsgemeinschaft, verglichen mit jenem, das ursprünglich die Androhung und hernach die Verfolgung und schließlich Vollstreckung von Kriminalstrafe leitet.

\section{BEHRENDS:}

Die nächste Wortmeldung darf ich mit einer besonderen Begrüßung verbinden. Herr Buback, Sie sind aus Gründen, die uns allen bekannt sind, unser Ehrengast und als Betroffener in besonderem Maße Ausdruck dessen, dass der Frage des strafenden Sozialstaats auch eine moralische und existentielle Dimension zukommt, die vom positiven Straf- und Strafprozessrecht nicht immer voll erfasst wird. Bitte, Herr Buback.

\section{BUBACK:}

Es freut mich, dass sich die Akademiekommission mit einer Thematik befasst, die eine Merkwürdigkeit berührt, mit der ich mich kürzlich konfrontiert sah, der fünffach lebenslangen Strafe. Die Vorstellung hat mich als Naturwissenschaftler verwirrt. Ich fand dann auch noch Verurteilungen zu einer „nur“ zweimal lebenslänglichen Strafe. Wie ich inzwischen gelernt habe, gibt es diese Strafen nicht mehr, was mich natürlich beruhigt.

Als Nichtjurist kann ich zum Thema nur wenig beitragen. Im vergangenen Jahr habe ich mich nur mit einigen kriminalistischen Dingen befasst, die dem Naturwissenschaftler viel näher stehen als Juristisches. Nach Kontakten mit Herrn Behrends ist mir aufgefallen, dass der Gerichtsvorsitzende, der heute eine lebenslängliche Strafe verkündet, sofort im Nachsatz sagen müsste, diese 
lebenslängliche Strafe dürfe aber keinesfalls lebenslänglich andauern. Weil das ja doch ein gewisser Widerspruch ist, sehe ich hier Klärungsbedarf und würde mich freuen, wenn die Kommission dazu beitragen könnte.

Ein zweiter Punkt betrifft den Mehrfachmord, bei dem die Strafandrohung nicht mehr angemessen sein könnte, wenn die Gesamtstrafe auf einen Zeitraum von beispielsweise 15 Jahren begrenzt wird. Diese Limitierung kann dazu führen, dass ein einzelner Mord gleichsam ungesühnt bleibt. Beim Massenmord konvergiert ja das Strafmaß für den einzelnen Mord gegen sehr kleine Haftdauern. Ich möchte die eventuelle Misslichkeit einer begrenzten Haftdauer an einem Beispiel illustrieren: Der Täter, der bei einem Banküberfall einen Sicherheitsbeamten ermordet, könnte sich aus nüchternem Kalkül dazu entschließen, auch noch Zeugen zu erschießen. Wenn die Zeugen ausgeschaltet wären, hätte das für ihn den Vorteil, später vielleicht nicht überführt zu werden, während kein oder kein wesentlicher Nachteil bei einer Verurteilung besteht, wenn sich die Haftdauer durch weitere Morde nicht erhöht.

Sie werden sich vielleicht über solche Gedankenspiele eines Naturwissenschaftlers wundern, aber es ist generell gut, bei den Regeln, die Naturwissenschaftler und Juristen, aber auch viele andere nutzen, jeweils zu prüfen, ob das System oder das Regelwerk auch für Grenzfälle vernünftige Lösungen bereit hält.

Der dritte Punkt betrifft die Wirkung von Strafen, bevor die Tat begangen wird. Es sollte eine Ausgewogenheit geben, indem eine angemessene Strafandrohung für diejenigen existiert, die das Leben anderer gering schätzen. Dies soll aber keinesfalls ein Argument für die Todesstrafe sein, die ich ablehne, schon deshalb, weil sie im Falle einer Fehlentscheidung nicht revidierbar ist.

Noch zwei kleinere Punkte: Sie betreffen die Möglichkeit einer Koppelung von vorzeitiger Freilassung durch Begnadigung mit einem Bekenntnis der Verurteilten zur Tat. Mir erscheint es richtig, im Falle einer Begnadigung zu wissen, für welche Tat sie gewährt wird. In diesem Sinne ist es bedauerlich, aber wohl nicht zu ändern, dass ein Täter seine Tat nicht einräumen muss.

Es würde mich noch interessieren, wie sich die Problematik in anderen europäischen Ländern darstellt. Es gibt im terroristischen Bereich den Fall, dass ein Täter in einem Land zu 20 Jahren Haft verurteilt wurde. Er wurde dann wegen einer anderen Straftat, für die ihm eine lebenslange Strafe drohte, ausgeliefert. Die Verurteilung zu lebenslänglich erfolgte, aber die tatsächliche Haftdauer lag dann unter 20 Jahren.

BEHRENDS:

Vielen Dank. Bedauerlicherweise sind wir in der Zeit doch schon ein wenig fortgeschritten. Ich möchte daher jetzt vorschlagen, dass wir nun doch dazu übergehen, die Fragen zu sammeln. Herr Müller-Dietz, Frau Hörnle und Herr Patzig sind noch auf der Rednerliste. Zunächst Herr Müller-Dietz. 
MÜLLER-DiETZ:

Ich habe ein paar Beiseitebemerkungen, die ich ganz kurz fassen möchte, und eine Frage an Herrn Duttge. Die Frage vorweg: Haben Sie sich deshalb auf den Tatausgleich und den Schuldausgleich konzentriert, weil Sie die Strafe als ein aliud gegen alle anderen Formen, wie sie Herr Naucke geschildert hat, von sozialen Interventionen abheben? Und nun zu meinen kleinen Beiseitebemerkungen: Eine betrifft das, was Herr Buback gesagt hat, das Problem des Zeitmaßes beim Mord: Wenn ein Mörder mit 70 Jahren den Mord begeht, dann hat es die Gerechtigkeit schwer. Das Problem, was Sie, Herr Duttge, angesprochen haben über die Maßregel: Es ist völlig klar, und so haben wir es früher auch gelehrt, dass die Maßregeln im Grunde Polizeirecht in allen Richtungen darstellen. Nur das Praxisbedürfnis ist besonders deutlich geworden in einem Fall, der in England gespielt hat. In England hat man nämlich, ich weiß nicht, ob das im Gesetz irgendwo eingegangen ist, zeitweilig jedenfalls die Frage diskutiert, ob man jemanden, der bei irgendeiner Begutachtung als für die Gesellschaft gefährlich erscheint, auf unbegrenzte Zeit unterbringen darf. Ohne Nachweis einer Straftat. Das macht deutlich, dass es hier nur um den Schutz der Gesellschaft und nicht um irgendeine Ahndung von Straftaten geht.

\section{BEHRENDS:}

Frau Hörnle.

\section{HÖRNLE:}

Eine Anmerkung zu den Überlegungen von Herrn Behrends, dass eigentlich auf einer ersten Ebene die Todesstrafe bei Mord die angemessene Sanktion wäre und erst auf einer zweiten Ebene dann der Humanismusgedanke ins Spiel kommt, der dazu führt, dass wir die Todesstrafe nicht verhängen. Ich bin mir nicht ganz sicher, ob diese Argumentation mich überzeugt. Denn die erste Ebene könnte man natürlich mit dem Gedanken angreifen, dass sie eigentlich nur plausibel zu machen ist, wenn man den Gedanken der absoluten Proportionalität, der spiegelnden Strafen zugrunde legt. An diesen Gedanken würde ich anknüpfen und sagen, hier wirkt natürlich auch, aber nicht nur der Humanismusgedanke, sondern schon der Grundgedanke, dass spiegelnde Strafen nicht funktionieren. Bei fast allen Delikten funktioniert spiegelndes Strafen nicht. Und bei Mord auch nicht. Was machen Sie denn als spiegelnde Strafe mit demjenigen, der mit einer Bombe 500 Leute zerfetzt?

\section{BEHRENDS:}

Vielen Dank. Ich beantworte das am Ende. Herr Patzig.

PATZIG:

Wenn man nun als ein Angehöriger eines anderen, wenn auch in gewisser Weise benachbarten Fachs das, was bisher vorgetragen worden ist, anhört, hat man natürlich erstens das Gefühl der Bewunderung der wahrgenommenen Kompetenz. Zweitens aber auch, und das liegt wohl auch an Zeitgründen, den Eindruck einer gewissen Abschirmung gegenüber der ja nun wirklich jahrtau- 
sendelangen Diskussion über Fragen des Strafrechts oder des Strafens, die in der Philosophie stattgefunden hat. Wenn man daran denkt, dass schon der erste Satz der griechischen Philosophie, den wir kennen, in dem ANAXIMANDER

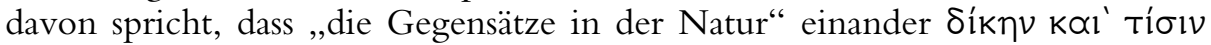

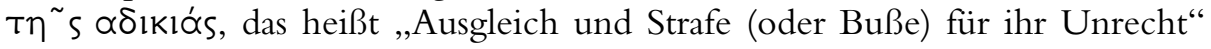
leisten, sieht man, dass die ganze Welt von den Griechen zu Anfang als eine Art von Kampf von Gerechtem und Ungerechtem oder Gutem und Bösem angesehen wird. Bei PLATON liest man, dass die Strafe für begangenes Unrecht deswegen verhängt werden muss, weil sie gut für die Seele des Bestraften ist, so als eine Art von Heilmittel. Der Grundsatz hat sich lange erhalten, es sei eigentlich auch im Interesse des Täters selbst, dass er bestraft wird, damit seine Seele von der Krankheit wieder gesundet. Und man denkt daran, dass KANT gesagt hat, ,,wenn die Menschheit sich einmal wie ein Verein auflösen würde, müsse vorher noch der letzte verurteilte Mörder hingerichtet werden - denn Recht muss Recht bleiben“".

Man sieht, dass in unserer geistigen Tradition das Schuldprinzip als Strafgrund sehr im Vordergrund steht. Es ist offenbar nach verbreiteter Auffassung immer eine Aufgabe der Gesellschaft gewesen, dafür zu sorgen, dass Schuld durch Strafe ausgeglichen wird. Es war schon eine gewisse Humanisierung, dass man diesen Ausgleich nicht den Opfern überließ, sondern der Allgemeinheit anvertraute, die dann in günstigen Fällen auch objektiver und vielleicht auch milder urteilt.

Nur einen weiteren Punkt will ich noch zur Erwägung stellen: Man kann sich doch darüber sehr wundern, dass eben nur eine bestimmte Sorte von Handlungen im Strafrecht verboten wird. Das Maß der Schuld, das jemand auf sich lädt, wird weithin daran gemessen, wie stark die Strafvorschriften in einem solchen Kodex sind. Dass es moralisch abscheuliche Handlungen gibt, die in ihrer menschlichen Niedertracht weit einen gewöhnlichen Erpressungsversuch, einen Diebstahl oder Banküberfall übersteigen, von denen aber unsere Gerichte aus verschiedenen Gründen keine Notiz nehmen und wohl auch gar keine Notiz nehmen können, ist doch offensichtlich. Was gibt es für Qualen, die Menschen aushalten müssen, weil sie zum Beispiel irgendjemanden in der Verwandtschaft, Familie, vielleicht Ehepartner haben, die es darauf anlegen, ihnen das Leben zur Hölle zu machen. Das wird im Allgemeinen nicht bestraft. Es gibt also nur eine gewisse Kategorie von Handlungen, die strafbar sind. Natürlich gibt es gute Erklärungen dafür; denn solche Verhaltensweisen kann man im Allgemeinen nicht nachweisen usw. Trotzdem meine ich, wenn man von Schuld spricht, sollte man nicht vergessen, dass juristische und rechtliche Schuld und moralische Schuld zwei ganz verschiedene, sich nur teilweise überlappende, aber nach meiner Meinung, was das menschliche Unglück an-

1 Immanuel Kant, Die Metaphysik der Sitten (1797), Akademie-Ausgabe, Bd. VI, S. 333. 
geht, vielleicht durchaus gleichberechtigte Kandidaten für eine Diskussion wären.

BEHRENDS:

Vielen Dank. Jetzt Herr Duttge.

DUTTGE:

Ich beginne in der umgekehrten Reihenfolge, nicht ohne zuvor aber allen, die sich zu Wort gemeldet haben, für die vielen weiterführenden Hinweise zu danken. Zu dem, was Sie, lieber Herr Patzig, beschrieben haben, sind mir sogleich auch noch die Vollzugsdefizite bei durchaus für strafbar erklärten Taten in den Sinn gekommen. Solche in manchen Deliktsbereichen erheblichen Vollzugsdefizite stellen natürlich ein weiteres Problem dar für ein Strafverständnis, das der verdienten Übelszufügung für eine schuldhaft begangene Tat verhaftet ist. $\mathrm{Zu}$ der von Ihnen betonten Bindung dessen, was bestraft wird, an dasjenige, was durch Strafgesetze zuvor für strafbar erklärt worden ist, und damit zwangsläufig unter Ausschluss anderer, nicht erfasster Taten, die ebenso verwerflich oder noch schlimmer erscheinen mögen, will ich nur anmerken: Der Grundsatz „,nullum crimen, nulla poena sine lege“ gilt uns seit FEUERBACH als „heilige“ Errungenschaft rechtsstaatlichen Strafrechtsdenkens - aber nicht nur wegen der sich mit der Strafandrohung verbindenden Hoffnung auf effektive Abschreckung aller potentiellen Delinquenten (das mag man eher für einen romantischen Gedanken halten), sondern in erster Linie deswegen, weil wir dem Täter bei vorheriger gesetzlicher Kennzeichnung des strafwürdigen Unrechts die Schutzbehauptung abschneiden, er habe sich infolge seiner Unkenntnis der Verbotsnormen gar nicht gegen das Recht aufgelehnt: „Error iuris nocet" - sofern nicht aufgrund besonderer Umstände ausnahmsweise unvermeidbar (vgl. \$ $17 \mathrm{StGB}$ )! Dasjenige, was der Gesetzgeber für strafbar erklärt, ist beschränkt auf die Verletzung oder Gefährdung von „Rechtsgütern“, um der Kriminalisierung bloßer Moralwidrigkeiten entgegenzuwirken. Letztere mögen, wie z.B. der Ehebruch, das seelische Befinden des Opfers in besonders schlimmer Weise verletzen; das gibt aber trotz des auch hier - noch dazu besonders gravierenden - Vertrauensbruchs noch keinen Grund, solches mit Kriminalstrafe $\mathrm{zu}$ ahnden. Das gilt um so mehr, wenn man im Lichte eines freiheitlichen Rechtsverständnisses die Begehung von Straftaten normativ nicht als Regelfall, sondern als Ausnahme ansieht: Strafrecht zielt auf dem Boden des Grundgesetzes auf einen „fragmentarischen“, ,subsidiären“ Rechtsgüterschutz und soll nur „ultima ratio" hoheitlicher Reaktion sein - oder anders gewendet: Von der fundamentalen Vorgabe des Art. 103 II GG ausgehend gibt es keine „Strafbarkeitslücken“, sondern allein „Straflosigkeitslücken“!

Zum Beitrag von Frau Hörnle, der sich auf die Todesstrafe bezog: Ja, das sehe ich ganz wie Sie. Die entscheidende Frage geht also dahin, was schon auf der ersten und auch einzigen "Stufe“ als schuldangemessen gelten kann für Verbrechen, die so schwer wiegen, dass sie in anderen Rechtsordnungen teilweise mit der Todesstrafe geahndet wurden oder selbst heute noch geahndet 
werden. Dass wir uns den Zugriff auf diese Sanktionsart kategorisch versagen, hat seinen Grund nicht in einer eigentlich inkonsequenten, gönnerhaften Milde und auch nicht - jedenfalls nicht nur - in den meist nicht restlos zu beseitigenden letzten Zweifeln am Nachweis der Tat, sondern hängt viel mehr damit zusammen, dass wir diese Sanktionsart als schlechthin unvereinbar mit unserem Rechtsverständnis ansehen. Und das wiederum hat unverkennbar sehr viel mit unserem Grundverständnis zur Reichweite legitimer Staatsgewalt im Verhältnis zu den rechtsunterworfenen Personen wie auch damit zu tun, dass wir insoweit wohl von einem Menschenbild ausgehen, das sich der lebensweltlichen und gesellschaftlichen Kontextualität allen Menschseins nicht gänzlich verschließt. Oder von der anderen Seite her betrachtet: Vom Extremfall des Massenmörders einmal abgesehen, wird man zur Beurteilung, dass Todesstrafe für Kapitaldelikte angemessen sein könnte, wohl nur dann kommen, wenn eine doch sehr individualistisch-idealistische, streng auf Eigenverantwortlichkeit abstellende Betrachtungsweise zugrunde gelegt wird nach der Devise: „Die Tat tötet den Mann" (oder die Frau). Ich kann an dieser Stelle also wiederum an dasjenige erinnern, was ich schon mehrfach betont habe.

Zur Frage von Herrn Müller-Dietz: Besteht zwischen Strafe und anderen hoheitlichen Sanktionsformen ein Aliud-Verhältnis? Ja, das ist ganz mein Verständnis und findet sich im geltenden Recht durchgängig angelegt. Es gilt dann, nicht nur bei den Maßregeln der Besserung und Sicherung, sondern auch gänzlich außerhalb des (formellen) Strafrechts, zur Begrenzung staatlicher Gewalt nicht das Schuldprinzip, sondern der Verhältnismäßigkeitsgrundsatz das ist etwas anderes, auch wenn das Bundesverfassungsgericht in manchen Entscheidungen missverständlich eine deutliche Nähe behauptet. Verhältnismäßigkeit bedeutet Proportionalität der Eingriffsintensität, das heißt, im Verhältnis zum betreffenden Gemeinwohlbelang. Dann kann es eben sein, dass bei fortbestehender hochgradiger Gefährlichkeit unter Umständen auch lebenslanger Freiheitsentzug als erforderlich und angemessen erscheint. Im Falle der Strafe besteht dagegen eine notwendige Korrelation mit der schuldhaft begangenen Tat, wie auch immer man diese Relationalität näher berechnen kann. Und diese ist nach der Rechtsprechung des Bundesverfassungsgerichts aus verfassungsrechtlichen Gründen stets so begrenzt, dass dem Täter nicht nur der physische, sondern auch der „soziale Tod“" regelmäßig erspart bleiben soll.

BEHRENDS:

Vielen Dank für diese wunderbar kurze Antwort. Ich will mich auch kurz fassen. Die Idee einer Spiegelung, Frau Hörnle, mag ich schon als Romanist nicht. Dieser Gedanke gehört in die deutschrechtliche Theorie der spiegelnden Strafen, einer zuspitzenden Variation des archaischen Talionsgedankens, des Auge um Auge, Zahn um Zahn. Mir geht es um das Verhältnis von Strafen im Sozialstaat und moralischem Wert des geschützten Lebens des Individuums. Moralisch gesehen ist ein Menschenleben des anderen wert. Für das geltende Recht zeigen das Notwehr und Nothilfe. Demgegenüber hat die Humanisie- 
rung der Strafe im Sozialstaat, so begrüßenswert sie ist, die Folge, dass die Strafe immer weniger in der Lage ist, das nach wie vor gültige moralische Unwerturteil auszuschöpfen und darzustellen. Hier hat sich eine Entwicklung vollzogen, die auch im Beitrag von Herrn Patzig anklang und über die nachgedacht werden sollte. Wenn die Sozialexistenz des Täters so heilig ist, dass, so schwer die Tat auch sein mag, sie am Ende doch irgendwie, zumindest am Horizont, sichtbar bleiben muss, wir kennen ja alle die Regelungen, wird es immer wieder Fälle geben, in denen das moralische Unwerturteil verletzt wird. Es ist auch nicht von der Hand zu weisen, dass der professionelle Umgang mit solchen Regelungen eine gewisse Unempfindlichkeit oder gar einen gewissen Zynismus in Strafrechtspflege und Strafrechtswissenschaft hinein tragen kann. 
